\title{
Where is Industry Getting it Wrong? A Review of Quality Concerns Raised at Day 120 by the Committee for Medicinal Products for Human Use during European Centralised Marketing Authorisation Submissions for Chemical Entity Medicinal Products
}

\author{
John Joseph Borg, ${ }^{\text {a, }} \mathbf{h}$ Jean-Louis Robert, ${ }^{\mathbf{b}, ~ h}$ George Wade, ${ }^{\text {c }}$ George Aislaitner, ${ }^{\mathbf{d}, \mathbf{h}}$ Michal Pirożynski, ${ }^{\mathbf{e}, \mathbf{h}}$ Eric Abadie, ${ }^{\mathbf{f}, \mathbf{j}}$ \\ Tomas Salmonson, ${ }^{\text {g, i }}$ Patricia Vella Bonanno, ${ }^{\mathbf{a}, \mathbf{h}}$ \\ ${ }^{\mathbf{a}}$ Medicines Authority, Gzira, Malta; ${ }^{\mathbf{b}}$ Laboratoire National de Santé, Luxembourg; ${ }^{\mathbf{c}}$ European Medicines Agency

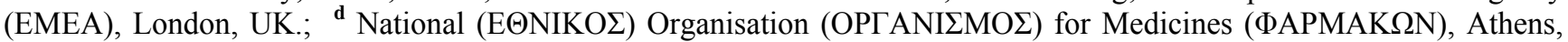 \\ Greece; ${ }^{\mathbf{e}}$ Department of Anaesthesiology and Critical Care Medicine, Postgraduate Medical School, Warsaw, Poland; \\ ${ }^{\mathbf{f}}$ Agence Française de Sécurité Sanitaire des Produits de Santé, Cedex, France; ${ }^{\mathrm{g}}$ Läkemedelsverket, Uppsala, Sweden; \\ ${ }^{\mathbf{h}}$ Member, ${ }^{\mathbf{i}}$ Vice Chair, ${ }^{\mathbf{j}}$ Chair of the Committee for Medicinal Products for Human Use, European Medicines Agency \\ (EMEA), London, UK.
}

Received, March 26, 2009; Revised, June 9, 2009; Accepted, July 14, 2009; Published, August 6, 2009

The views expressed in this article are the personal views of the authors and may not be used or quoted as being made on behalf of, or reflecting the position of, any national competent authority, the EMEA or one of its committees or working parties.

\begin{abstract}
Purpose. The aim of this study was to identify common trends in the deficiencies identified in the quality part of the dossier during the evaluation of marketing authorisation applications for medicinal products for human use submitted through the EU's centralised procedure. Methods. We analysed all the adopted Day 120 list of questions on the quality module of 52 marketing authorisation applications for chemical entity medicinal products submitted to the European Medicines Agency and evaluated by the Committee for Medicinal Products for Human Use (CHMP), during 12 consecutive plenary meetings held in 2007 and 2008. Subsequently we calculated the frequency of common deficiencies identified across these applications. Results. Frequencies and trends on quality deficiencies have been recorded and presented for 52 marketing authorisation applications. 32 "Major Objections" originated from 13 marketing authorisation applications. 13 concerned were raised regarding drug substances and 19 for drug products. Furthermore, 905 concerns on drug substance and 1,054 on drug product were also adopted. Conclusions. The impact of the frequencies and trends in quality deficiencies that were identified are discussed from a regulatory point of view. It is expected that the results of this study will not only be of interest to pharmaceutical companies but will also aid regulators' in obtaining consistent information on drug products based on transparent rules safeguarding the necessary pharmaceutical quality of medicinal products.
\end{abstract}

\section{INTRODUCTION}

In order to obtain a marketing authorisation by the European Commission for a product to be simultaneously valid in all member states of the European Union and recognised by the states of the European Economic Area (EEA), an application must be submitted through the centralised procedure to the European Medicines Agency (EMEA). This marketing authorisation procedure for medicinal products for human use is currently governed by Regulation (EC) 726/2004 (1), and the marketing authorisation granted by the European Commission is based on a scientific opinion from the EMEA's Committee for Medicinal Products for Human Use (CHMP). The annex of the abovementioned regulation defines the types of product that must obtain a marketing authorisation solely through the centralised procedure and prospective applicants have no other choice. In addition to the annex, certain medicinal products which contain a new active substance or which can be shown to constitute a significant scientific, technical or therapeutic innovation, even if they are generics,

Correspondence author: Dr. John Joseph Borg, Medicines Authority, 203 Level 3, Rue D’Argens, Gzira, GZR 1368, Malta. john-joseph.borg@gov.mt 
may optionally be submitted to the EMEA and will be accepted if judged to be eligible by the CHMP.

In the process of issuing an opinion for a marketing authorisation application (MAA), the CHMP (or more precisely the (Co)-rapporteurs (rapporteur and co-rapporteur) assessment teams) carries out the scientific evaluation of the dossier on the medicinal product provided by the applicant. During the scientific evaluation of an applicant's common technical document (CTD) $(2,3)$, concerns are identified and forwarded to the applicant to resolve, prior to adopting an opinion on the medicinal product's risk-benefit profile. Specifically, on Day 120, the CHMP will adopt a list of questions outlining major and minor concerns on quality, safety and efficacy that must be addressed before granting of the marketing authorisation.

Within an assessment report, the terminology 'major objection' has been chosen for a serious deficiency which are either unresolvable or must be resolved by the applicant, whereas the term 'other concerns' is used to identify those deficiencies which need to be addressed and may not necessarily be regarded as a barrier to subsequent authorisation of the product.

This study reviews the adopted Day 120 list of questions on the quality assessment of medicinal products containing active substances which are small-molecule chemical entities (i.e. not biologicals), as reviewed by the CHMP during 12 consecutive plenary meetings held in 2007 and 2008. It was decided to utilise the Day 120 list of questions since this list represents the conclusions of the first consolidated review of the data included in the dossier, as endorsed by the CHMP. As a consequence, this list of questions contains the majority of the objections, concerns, and general deficiencies which in the course of the procedure must be resolved depending on the level of seriousness, in order to lead to a marketing authorisation.

In order to identify common/general trends in the quality deficiencies, we analysed all adopted CHMP Day 120 questions on the quality module (module 3) of 52 MAAs to calculate the frequency of common deficiencies identified across these MAAs. The results indicate that some MAAs submitted for evaluation contained documentation that lacked a systematic approach that were clear enough for evaluators to understand the manufacturing process and drug product. Herein a discussion is presented on the need for improving the adequacy of guidelines (ICH and/or CHMP notes for guidance) on quality of pharmaceuticals for human use and the European Commission's Notice to Applicants.

\section{METHODS AND MATERIALS}

\section{Creation and analysis of a database on quality concerns}

Adopted Day 120 list of questions for 52 MAAs of chemical medicinal products by the CHMP during 12 consecutive plenary meetings held in 2007 and 2008 were used to create a database of questions on quality deficiencies. The numbers and types of MAAs reviewed are included in Table 1. All Major Objections adopted by the CHMP on module 3 were sorted per section of the CTD. They were all related to drug substance or drug product. The Major Objections were analysed and objections raised on a specific section of module 3 were then grouped together and trends reported. No descriptive statistics were calculated as the numbers of Major Objections were too few to provide robust inferences. On the other hand, all other concerns (deficiencies in the CTD that need to be addressed during the evaluation of the MAA) adopted on module 3 of the CTD $(2,3)$ were sorted and grouped according to the specific subsection of the quality dossier. The module 3 subsections are listed as follows 3.2.S.1 - General Information; 3.2.S.2 Manufacture; 3.2.S.3 - Characterisation; 3.2.S.4 Control of Drug Substance; 3.2.S.5 - Reference Standards or Materials; 3.2.S.6 - Container Closure System; 3.2.S.7 - Stability; 3.2.P.1 - Description and Composition of the Drug Product; 3.2.P.2 Pharmaceutical Development; 3.2.P.3 Manufacture; 3.2.P.4 - Control of Excipients; 3.2.P.5 - Control of Drug Product; 3.2.P.6 Reference Standards or Materials; 3.2.P.7 Container Closure System; 3.2.P.8- Stability.

Subsequently, the questions were then reviewed, and the absolute frequency (f) with which a concern was listed per section of the CTD reported. The percentage frequency of total concerns per section of CTD of common deficiencies identified from 52 marketing authorisation were calculated as follows: 
Percentage Frequency of concerns identified $=$

$\left\{\frac{\text { Frequency of specific concern adopted }}{\text { Total number of concerns adopted per section of CTD }}\right\} * 100$

(1)

Data are presented as nominal values. All charts were drawn and analysis carried out with Microsoft Office Excel 2003 (Microsoft Corporation, USA).

\section{RESULTS}

\section{Descriptive statistics on adopted Day 120 list of questions by the CHMP}

During the evaluation procedure of 52 MAAs, the CHMP raised a total of 1,991 concerns at Day 120 on the quality module of the applications. From these there where, 32 Major Objections originating from 13 applications, 13 concerned drug substance and 19 drug product. With regard to the minor concerns, they were, 905 concerns on drug substance and 1,054 on drug product. The results indicate that there is a nearly $1: 1$ distribution between the questions raised on drug substance and drug product. The average number of questions adopted during a MAA (not line-extensions $n=47$ ) were 40 and for line extensions $(n=5) \quad 18$, suggesting as expected, that for a line extension application which is based on an already approved medicinal product, most quality concerns would have already been resolved during the initial MAA and prior to the submission of the line extension application.

\section{Analysis of Major Objections adopted by the CHMP at Day 120 for 52 marketing authorisation applications}

Thirteen Major Objections on drug substance were adopted by the CHMP. No Major Objections on drug substance were raised during evaluation of line extension applications. This is logical, as concerns would have been addressed in the original application. Analysis of these Major Objections on drug substance, show trends in the objections raised with respect to: qualification and profiling of impurities, drug substance specifications, specifications for potential genotoxic impurities, stability and starting materials for active pharmaceutical ingredients. With respect to drug product, 19 Major Objections were raised on pharmaceutical development, stability, control of drug product, validation of the manufacturing process, container closure integrity and batch analysis. Tables 2 and 3 present descriptive summaries of the Major Objections adopted by the CHMP.

\section{Analysis of other concerns adopted by the CHMP at Day 120 for 52 marketing authorisation applications}

Figure 1 depicts the percentage frequency of other concerns adopted by the CHMP at Day 120 on drug substance (3.2.S) and drug product (3.2.P) respectively. The frequency of concerns raised by the CHMP result in the following percentage frequency sequence for drug substance (Figure 1, top): 3.2.S.4 (control of drug substance) > 3.2.S.2 (manufacture) $>$ 3.2.S.7 (stability) $>$ 3.2.S.3 (characterisation of drug substance) $>$ 3.2.S.6 (container closure system) $\approx$ 3.2.S.5 (reference standards and materials) > 3.2.S.1 (general information).

For drug product, the following percentage frequency sequence has been calculated (see Figure 2, bottom): 3.2.P.5 (control of drug product) > 3.2.P.3 (manufacture) > 3.2.P.8 (stability) > 3.2.P.2 (pharmaceutical development) > 3.2.P.4 (control of excipients) > 3.2.P.7 (container closure system) > 3.2.P.1 (description and composition) > 3.2.P.6 (reference standards or materials). The observations show that there are similarities in the percentage frequency of concerns identified between drug substance and drug product. Where most concerns raised by the committee were on control of drug substance and drug product ( $>30 \%$ for both 3.2.S.4 and 3.2.P.5), followed by concerns on the manufacturing ( $>20 \%$ for both 3.2.S.2 and 3.2.P.3) and stability ( $>10 \%$ for both 3.2.S.7 and 3.2.P.8). In order to explore further where the issues and concerns arose during the scientific evaluation of the MAAs, all adopted concerns were analysed and grouped together according to the section of the CTD to which they refer. The results are presented in Tables 4 and 5 and show the percentage frequency of identified concerns greater than $7 \%$ per section of the CTD. We opted not to present concerns that were less than $7 \%$ of the total concerns raised per section of the CTD by the CHMP as in our opinion, these concerns are rarely raised and do not impact the primary objective of this study.

Further analysis of the results obtained on drug substance (see Table 4) show that for control 

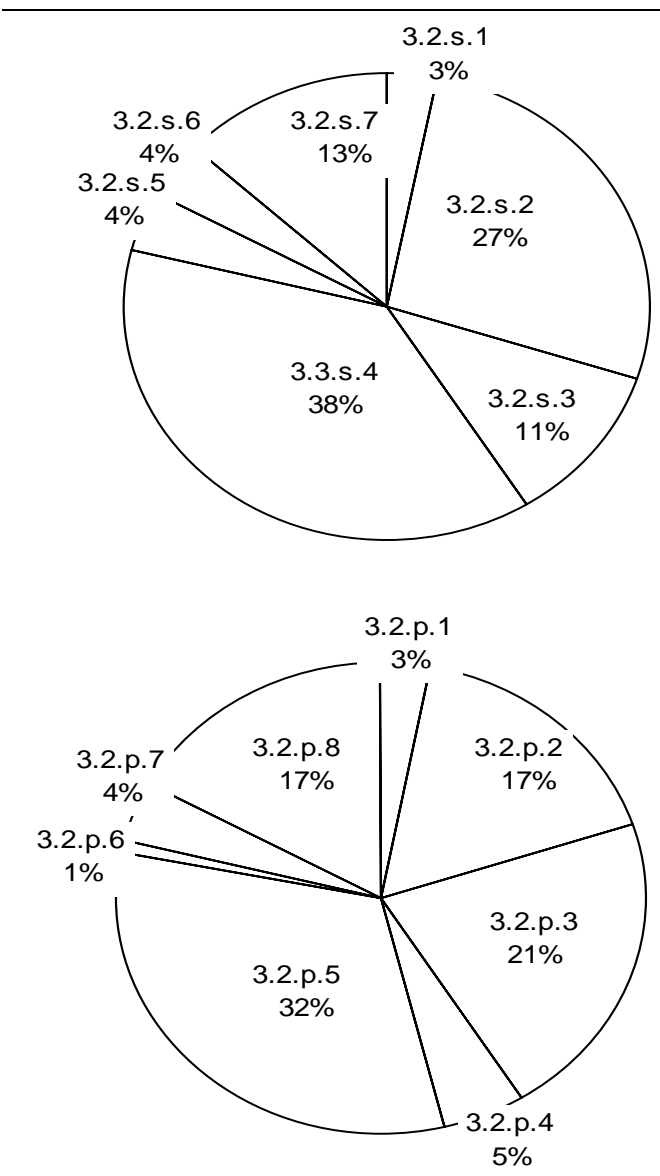

Figure 1. Percentage frequency of quality minor concerns adopted on drug substances (top, total number of questions adopted, 905) and drug products (bottom, total number of questions adopted, 1054) at Day 120 by CHMP for Marketing Authorization Applications of small chemical entities during April 2007 until May 2008. Labels denote the different sections of quality part (module 3) of the Common Technical Document $(2,3)$.

of drug substance (3.2.S.4), $65 \%$ of concerns were due to 1) the lack of specifications of drug substance; 2) the lack of a justification for specifications set and 3) the lack of analytical validation information relating to experimental data for the analytical procedures used for testing the drug substance. For manufacture of drug substance (3.2.S.2), $40 \%$ of deficiencies concerned: 1) specifications of intermediates and impurity profiles; 2) certificates of analysis (CoA) about the reference standards used to control the active substance as well as critical steps of manufacture and 3) details regarding the description of manufacturing process and process controls. For stability (3.2.S.7), 32\% of concerns focused on storage conditions and the retest period, where proposed storage conditions were not defined according to the ICH guideline on stability $(4,5)$ and the proposed retest period not acceptable in line with the ICH guideline on evaluation of stability data $(6,7)$. For characterisation of drug substance (3.2.S.3), 53\% of concerns were due to 1) lack of information on impurities that result from degradation, 2) lack of adequate discussion on the acceptability of a starting material containing an "alerting structure" in terms of genotoxicity and 3) lack of a discussion on whether starting materials contribute to the impurities of the drug substance.

With respect to the container closure system (3.2.S.6), 65\% of deficiencies regarded the quality of the primary packaging material for the active substance and the lack of information on the dimensions of all possible primary packages. For reference standards and materials (3.2.S.5), 84\% of all concerns related to the description of reference standards for impurities, the certificates of analysis of the reference standards used for the validation of the analytical methods and the lack of information for reference standard of known impurities. While for the section on the general information of drug substance (3.2.S.1), $62 \%$ of deficiencies were on the lack of information on physicochemical and other relevant properties of the drug substance such as light sensitivity, solubility, crystallization, polymorphism, batch size etc.

Analysis of the results obtained on drug product (refer to Table 5), show that for control of drug product (3.2.P.6), 30\% of concerns lay on issues relating to validation of analytical procedures and analytical methods (examples include: the HPLC method for related substances lacks data on Limit on Qualification and Detection for specified impurities, data on linearity/robustness/accuracy/ precision are lacking, selectivity of the method for identifying degradants needs to be discussed further). While for manufacturing (3.2.P.3), 19\% of deficiencies concerned lack of documentation supplied by pharmaceutical companies with respect to the description (details such as holding times of granules, intermediate products and bulk ware were lacking) of the manufacturing process. Furthermore, results of validation and/or evaluation studies should have been provided for critical steps or critical assays used in the manufacturing process (this includes production scale manufacturing at site of manufacture).

With respect to stability (3.2.P.8), 32\% of concerns regarded lack of data submitted by the applicant to substantiate the proposed shelf-life of the drug product (due to insufficient length of 
storage) and the lack of information on the analytical procedures used to generate stability data and validation of these procedures. For pharmaceutical development (3.2.P.2), $16 \%$ of concerns had to do with the results from comparative in vitro studies (for example the dissolution) or comparative in vivo studies (e.g., bioequivalence) requiring further discussion as well as a lack of information on the discriminatory power of dissolution method used. On the control of excipients $40 \%$ of concerns relate to certificates of analysis not being submitted and detailed specifications for each excipient not being set by the applicants. For the container closure system (3.2.P.7), $69 \%$ of concerns focused on requests to provide more detailed information regarding the packaging materials for the drug product ascertaining compliance with European Pharmacopoeia Monograph 3.1.4 (8) or Directive 2002/72/EC (9). While for description and composition of drug product (3.2.P.1), $46 \%$ of concerns related to the information on the composition of drug product. For reference standards or materials (3.2.P.6), $69 \%$ of concerns requested more information on the reference standards or reference materials used for testing of the drug product.

\section{DISCUSSION}

The results obtained show that common deficiencies have been identified by the CHMP during the scientific evaluation of MAAs. For both Major Objections and Other Concerns on the drug substance, most deficiencies identified across MAAs submitted by numerous diverse and different size pharmaceutical companies were related to the proposed specifications in the control of the drug substance and the specification, possible qualification and profiling of impurities (includes genotoxic impurities) and stability of the drug substance. These trends parallel the top 5 deficiencies published by the European Directorate for the Quality of medicines during the first assessment of applications for certificates of suitability from October 2007 and December 2007 (10). Comparing our results to the results of a study conducted in the early 90s analysing quality deficiencies from applications submitted to the CPMP (11), we report a shift from the problem areas identified (assays and limits, impurities, controls, manufacture of the active substance, development chemistry, batch analysis, and reference materials) by Jeffers and colleagues (11). With respect to the drug product, the most prevalent concerns common to both Major Objections and Other Concerns are related to pharmaceutical development and stability. Similarly, comparing our results to the problem areas identified by Jeffers and colleagues (11) on drug product (such as identity tests for active ingredients and excipients, method validation, batch analysis, assay limit justification, preservative limits, dissolution tests and limits, impurity degradation product and related substances controls, microbial contamination, and the limits proposed for the release and shelf life specifications), we report a shift in the trends in deficiencies nowadays by the CHMP. Perhaps these shifts in deficiencies identified for active substance and drug product are not entirely unexpected since the state of the art on the techniques and regulatory requirements have changed since the early 90 s.

The development and registration of a medicinal product is complex, where regulatory authorities and the pharmaceutical industry share a common goal of protecting public health without inhibiting the free movement of goods within the European market. It is therefore, in the best interest of all stakeholders to add to the available armamentarium in the diagnosis and treatment of diseases and pathological conditions in the shortest possible time. One way in which this is possible is for applicants to submit high quality documentation that can support the granting of a marketing authorisation without Major Objections and/or numerous concerns being identified. Analysis of the results obtained from this study show that there exist trends in the quality concerns identified and raised by the CHMP. This prompts a question: Does the trend reflect the scarcity of guidelines or the existence of too many subsequent guidance resulting in misinterpretations? The answer to the above questions is not clear cut. However, most ( $>$ $70 \%$ ) of the concerns identified with respect to the lack of information supplied by applicants on both the drug substance and drug product could have been avoided if the guidance in the ICH CTD (12) and "Notice to Applicants Vol. 2B" as published by the European Commission was followed by pharmaceutical companies (13). On the other hand, some concerns raised could be avoided if applicants had been made aware of the following issues that are being raised during the assessment of centralised MAAs: details for holding times of 
intermediates during the manufacturing process should be submitted by applicants, detailed specifications in control of drug substance and product and possible impurities should be submitted by applicants, the quantification of related substances; the amount of data that needs to be presented with respect to dissolution of tablets.

Another plausible answer to the above question may be that the quality assessors in the EU focus on the specifications of the active substance and on product development in order to guarantee a tight quality control of the drug substance and or product. Whatever the reasons for the results in this paper, in the absence of any procedures set by the EMEA to improve the quality documentation of centralised marketing authorisation applications, companies would do well to pay attention to the findings to put their dossiers in the best possible state of completion in anticipation of a rigorous assessment in the areas identified as generating large numbers of questions.

To improve the quality of submissions for both new and abridged new drug applications, perhaps, the EMEA/CHMP should take into account the U.S. FDA's approach. The latter agency includes in the process, a question based review system with a defined set of questions which have to be addressed in the module 2.3 Quality Overall Summary (QOS). Subsequently, the responses to the quality based review questions are documented in the QOS but derived from all the data included in Module 3. Any steps taken in the future by the EMEA/CHMP in order to improve the quality of applications submitted should be monitored on an ongoing basis and their effectiveness in improving the quality of documents submitted captured through the peer review procedure that has recently been introduced by the EMEA during the assessment of MAAs.

It should be kept in mind that this study is a snap shot of the review of the deficiencies/objections adopted by the CHMP during the review of 52 Marketing authorisations at Day 120 of the centralised procedure and most of these applications are still under review during submission of this paper. Therefore, we have a shortcoming that we are not able to verify if these objections and concerns raised have resulted in a delay in the granting of a marketing authorisation. However, a review of the European Public Assessment Reports (EPARs) available on the EMEA's website indicates that quality objections for chemical entities rarely result in the refusal of the granting of marketing authorisation. In practice, the most likely finding is that Major Objections in the area of quality are indeed resolvable in the time allowed before the end of the procedure, but additional time may be needed at the discretion of CHMP, resulting in delays. The CHMP also has the option to introduce another round of questions later in the procedure - a list of issues - with a stop clock and further delay. Residual quality concerns which are not resolved at the end may be set aside for post-opinion/post-authorisation resolution if, in the opinion of CHMP, it is considered they are so minor that they have no significant impact on the benefit-risk balance of the product. However, this last option does lock the company into quite complicated and tedious post-authorisation commitments in the area of quality.

To the best of our knowledge, this is the first study carried out on quality deficiencies raised during the scientific evaluation of MAAs by the CHMP since Regulation 726/2004/EC became effective. Our intention has been to reduce unnecessary and avoidable delays in otherwise safe and effective medicines reaching the EU market, and ultimately this will be of benefit to patients.

Table 1. Type of Marketing Authorisation Application

\begin{tabular}{lc}
\hline Type & Number \\
\hline New active substances & 33 \\
Generics & 9 \\
Line extensions & 5 \\
Orphan medicinal products & 5 \\
Chiral Drugs & 8 \\
\hline
\end{tabular}



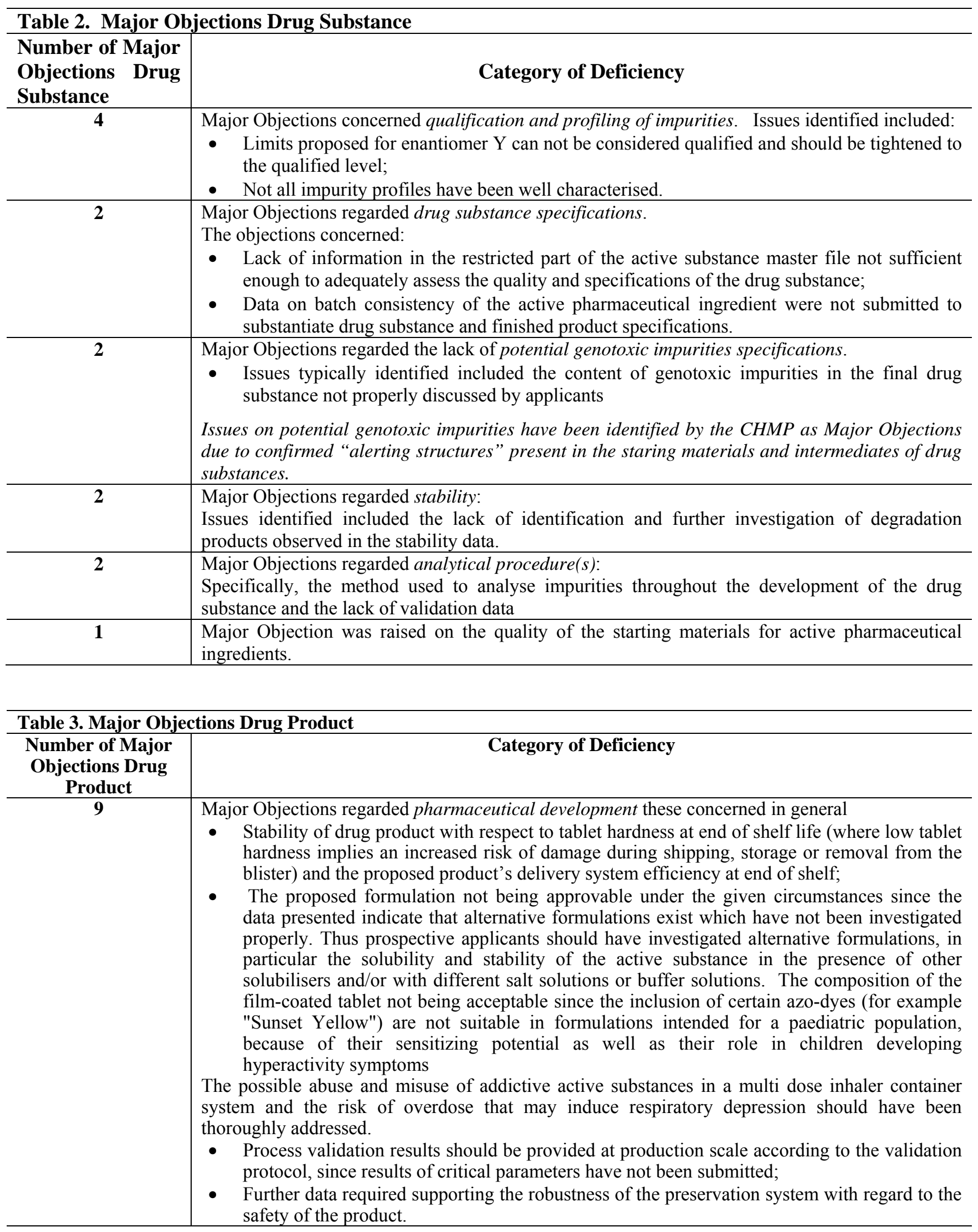

Continued... 


\begin{tabular}{c|l}
\hline Table 3. Major Objections Drug Product, ..... Continued \\
\hline $\mathbf{4}$ & $\begin{array}{l}\text { Major Objections regarded the stability of drug product, } \\
\text { Issues identified included: } \\
\text { The data regarding the stability of the drug product are not plausible and, therefore, not } \\
\text { reliable; }\end{array}$ \\
\hline $\mathbf{A}$ & $\begin{array}{l}\text { Issues regarding colour development not being fully justified by applicants during storage; } \\
\text { Significant decrease in the assay values for finished product stored in containers at } \\
\text { accelerated conditions. }\end{array}$ \\
\hline $\mathbf{2}$ & $\begin{array}{l}\text { Major Objections regarded the control of drug product, specifically on specifications not covering } \\
\text { drug product sterility adequately. }\end{array}$ \\
\hline $\mathbf{2}$ & $\begin{array}{l}\text { Major Objections regarded the validation of a non-standard manufacturing process of a drug } \\
\text { product not being adequately carried out applicants. }\end{array}$ \\
$\begin{array}{l}\text { 1 Major Objection concerned the container closure integrity not being properly documented and } \\
\text { another Major Objection concerned peaks above the identification limit in the batch results for } \\
\text { batches used for clinical trials not being identified by the applicant. }\end{array}$ \\
\hline
\end{tabular}

Table 4. Other Concerns Drug Substance

\section{Category of deficiency}

MODULE 3.2.S.1 - General Information

- Physicochemical and other relevant properties of the drug substance such as light sensitivity, solubility, crystallization, polymorphism and batch size should be provided;

- Different forms mentioned in section 3.2.S.3.1 of the CTD should be described.

- The structural formula, including relative and absolute stereochemistry (isomerism), the molecular formula, and the relative molecular mass should be provided in the dossier;

- Information about the manufacturer provided should correspond to current manufacturer, manufacturing site and manufacturing procedure, all information in the dossier needs to be consistent.

Other concerns

MODULE 3.2.S.2 - Manufacture

- The specification of intermediates and impurity profile should be provided in the dossier;

- The possibility of the presence and/or formation of impurities in intermediates need to be justified by applicants; the proposed maximum batch size for each intermediate should be supported by data from the validation process;

- Confirmation of absence of class 1 or 2 solvents should be submitted by the applicants in the dossiers;

- Certificates of analysis (CoA) about the reference standards used to control the active substance need to be submitted;

- Critical steps should be identified, all limits proposed by the applicant need to be justified according to CPMP/QWP/130/96 (14).

\begin{tabular}{|c|c|}
\hline $\begin{array}{l}\text { Absolute } \\
\text { Frequency } \\
\text { (f) }\end{array}$ & $\begin{array}{c}\text { \% Frequency } \\
\text { of concerns/ } \\
\text { specific CTD } \\
\text { module }\end{array}$ \\
\hline
\end{tabular}

18

62

4

14

14

20

Continued ... 
Table 4. Other Concerns Drug Substance, .... Continued

- Description of manufacturing process and process controls (name, manufacturer, batch size etc) need to be stated in the dossier;

- Details of the test methods used by the manufacturers should be confirmed and appropriate validation data should be provided by applicants (this also includes tests in the active pharmaceutical ingredient (API) specification or intermediate specifications carried out before blending of final API batches or intermediates);

- Information on batches presented in section S.4.4 of the CTD lack detail on which batches have been prepared from blended intermediates and which have been reprocessed;

- The dossier lacks information of the synthesis in the form of a flow diagram.

- Materials used in the manufacture of the drug substance (e.g., raw materials, starting materials, solvents, reagents, catalysts) should be listed and presented in the dossier so that it is identified where each material is used in the manufacturing process;

- Information on the quality and control of these materials should be provided by the applicant;

- Information demonstrating that materials (including biologically-sourced materials, e.g., media components, monoclonal antibodies, enzymes) meet standards appropriate for their intended use (including the clearance or control of adventitious agents) should be present in the dossier;

- Applicants should state in the dossier which solvent(s) have been used in batches of the starting material.

- Information in the description of the manufacturing process in section 3.2.S.2.6 of the CTD and the process as described in section 3.2.S.2.2 of the CTD need to be consistent;

- Applicants should justify that all information regarding process development is incorporated in the process validation report and is submitted in the dossier;

- Applicants should describe and discuss any significant changes made to the manufacturing process and/or manufacturing site of the active substance used in producing non-clinical, clinical, scale-up, pilot, and production batches (this information is part of the restricted part of the active substance master file);

- The maximum batch size for which the applicant has experience with and the defined method used for production of the drug substance should be stated in the dossier.

- Applicants should submit information of the suppliers of the starting materials;

- Certificate of Analysis (CoA) should be supplied and need to comply with the European Pharmacopoeia;

- A declaration on the non-use of material of animal origin, susceptible to concern by TSE contamination, during the manufacturing process needs to be present in the dossier. 


\section{Table 4. Other Concerns Drug Substance, .... Continued}

MODULE 3.2.S.3 - Characterisation

Absolute

(f)
$\%$ Frequency

of concerns/

specific CTD

module

- Module 3.2.S.3.2 of the CTD should be updated with all impurities that result from degradation;

- Applicants should comment on the acceptability of a starting material that show a chemical group that maybe an "alerting structure" in terms of genotoxicity and the guideline "Limits of genotoxic impurities";

- Information presented regarding impurity profiles is not sufficient to show that no significant differences exist between the impurity profiles of the batches produced using different purification / isolation processes;

- Applicants should discuss if starting materials contribute to impurities;

- Data in section 3.2.S.3.2 of the CTD should be consistent with the batch results presented in section 3.2.S.4.4 of the CTD;

- Structural characterisation data and the method of preparation of all specified impurities need to be included in the dossier;

- Applicants should take all necessary steps to identify impurities and their strategy discussed in the dossier.

- Elucidation of structure and other characteristics should be presented in the dossier;

- Data and spectra of structure confirmation need to be supplied;

- Analytical methods used should be described and validated and data and spectra of structure confirmation supplied in the dossier.

- The analytical procedures used for analysis of impurities throughout the development of the medicinal product and their validation (including considerations on response factors) should be provided.

Other concerns

\section{6}

53

3

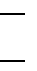


Table 4. Other Concerns Drug Substance, .... Continued

- identified impurities and specified unidentified organic impurities presented in section 3.2.S.3.2.1 and in section 3.2.S.4.5.1 of the CTD, the lack of a microbiological limit test in a substance intended for a parenteral product, the exclusion of a specific rotation specification, the content of residual

- $\quad$ organic solvents (for example more than $10 \%$ of ICH Q3C limit $(16,17)$ );

- Applicant should establish one common binding specification for active substance from both suppliers in accordance with the requirements of the European Pharmacopeia.

- Analytical validation information including experimental data for the

$\bullet 77 \quad \bullet 21$
analytical procedures used for testing the drug substance (accuracy, precision, specificity, quantitation limits, and linearities) should be provided in the dossier;

- Methods used and batches subjected for control of impurities should be provided;

- Validation reports for the analytical procedures used in the specification of the starting material and names and addresses of the suppliers (manufacturers) of the starting material are lacking in the dossier;

- Data on the accuracy of the related substances test is unsatisfactory with the average percent recoveries needing to be reported in the documentation submitted.

- Analytical procedures used for testing drug substance should be provided;

63

17

- Analytical results should be presented for commercial scale batches;

- Applicants should confirm that variation applications will be submitted if the analytical method is altered;

- Confirmation needs to be submitted that the proposed related substances methods used are the same as those used for the clinical and non-clinical batches manufactured;

- Analytical results should be presented for at least three batches with the level of impurities reported.

- Description of batches and results of batch analyses should be provided as well as their certificates of analysis to confirm the quality of the materials used from each active substance manufacturer;

- Comprehensive analysis data on three full-scale batches should be provided including the batch size and the manufacturing date;

- Data on batch-to-batch consistency must be provided in the dossier;

- Section 3.2.S.2.6 of the CTD must be updated with the development of analytical methods;

- Commercial batches should be tested in accordance to active substance specification in 3.2.S.4.1 of the CTD;

- Applicants should present data on influence on drug substance quality (e.g. level of organic impurities and other relevant parameter) by use of optional manufacturing procedures to be applied to the drug substance.

\section{MODULE 3.2.S.5 - Reference Standards or Materials}

- The reference standards for the impurities should be described. 11 30

- Certificates of analysis of the reference standards used for the validation of 10 27 the analytical methods should be provided. 


\section{Table 4. Other Concerns Drug Substance, .... Continued}

\section{Absolute \\ Frequency}

(f)

- Information for reference standards of known impurities is missing in the dossier;

- Reference standards of all specified and identified related substances as well as for all identified related substances used in validation of analytical methods for impurities and chiral purity should be characterised sufficiently and data provided in the dossier.

Other concerns
\% Frequency of concerns/ specific CTD module 27

\section{MODULE 3.2.S.6 - Container Closure System}

- Quality of the primary packaging material for the active substance should be documented in accordance with the requirements of the guideline on plastic immediate materials CPMP/QWP/4359/03 (18);

- Packaging material quality should be documented by a representative certificate of analysis and IR spectrum.

- The dimensions of all possible primary packages should be presented; 25

- Specification for the polyethylene bags should be provided that includes description and identification.

- Polyethylene used should comply with the European Pharmacopoeia monograph on Polyolefines (10)

- A detailed description of the container and closures used should be given in the dossier;

- Compliance with pharmacopoeial standards and EU directives should be demonstrated by applicants;

- The method of closure should be described in the dossier;

- Adequate protection from microbial contamination should be demonstrated.

Total of other concerns

\begin{tabular}{l|c}
10 & 27 \\
& \\
& 16
\end{tabular}

10

25

$7 \quad 18$

$5 \quad 13$

\section{MODULE 3.2.S.7 - Stability}

- Storage conditions and retest period have not been submitted by applicants;

- The proposed storage conditions should be defined according to ICH Q1E (evaluation of stability data) $(8,9)$ and the guideline on stability testing: stability testing of existing active substances and related finished products CPMP/QWP/122/02 rev 1 corr (19);

- At least three commercial batches should be tested according to the conditions required by ICH Q1A $(4,5)$ and according to the stabilityindicating parameters of the revised specification;

- In order to approve of a re-test period data are needed for relevant degradants that were not considered by the applicant;

- Results of ongoing studies should be provided in order to justify the proposed retest period and storage conditions;

- Relevance of earlier "registration stability study" carried out by the applicant on drug substance should be proved with information on manufacturing process or impurities (i.e. residual solvents, polymorphic feature of the batches tested) being provided. 
Table 4. Other Concerns Drug Substance, .... Continued

- Stability studies in the applicant's part of the active substance manufacturer file (ASMF) received from the drug substance manufacturer (ASM) need to be complete and the ASM should update this information;

- Stress stability studies should be detailed and discussed with potential degradation pathways and products;

- Evidence from studies conducted under stressed conditions should be provided to demonstrate that analytical methods used in stability trials are stability indicating; manufacturing date and batch sizes should be reported for all batches used during stability studies (information should include the batch size);

- Detailed analytical methods references and standards (lots) used should be provided in the dossier; analytical certificates of active pharmaceutical ingredient batches included in stability studies should be provided in the dossier.

- Any adverse findings of stability data covering the proposed re-test period should be reported to the EMEA;

1312

- A retest period is acceptable if company commits to communicate to the authorities any out of specification results on the first three production batches;

- A commitment that batches for long-term studies will be tested annually should be provided by applicants.

- Stability data from the batches manufactured recently should be presented to support the expiry claim;

- Confirmation that ongoing stability testing of the commercial batches will be performed in accordance with the drug substance specification should be submitted;

- Data supporting potential genotoxic impurities below the TTC limit in the batches placed on formal stability studies should be submitted.

- Discrepancies between assay levels of active substance and impurity level rising during stability studies need to be discussed by the applicant;

- Data that the active ingredients are present in satisfactory amounts at the end of stability trials should be provided by applicants.

- Data on all impurities monitored in stability studies should be provided in the dossier;

- Explanations as to why an impurity increases over a period of time should be supplied;

- Organic impurity results provided at release as well as stability results would suggest lowering the acceptance limit for total impurities;

- Applicant's should disclose if degradation products appear or not during forced degradation studies and if they are different or not than those listed under the impurities section of the dossier. 
Table 5. Other Concerns Drug Product

Category of deficiency

\begin{tabular}{cc}
$\begin{array}{c}\text { Absolute } \\
\text { Frequency } \\
\text { (f) }\end{array}$ & $\begin{array}{c}\% \text { Frequency } \\
\text { of concerns/ } \\
\text { specific CTD } \\
\text { module }\end{array}$ \\
\hline
\end{tabular}

MODULE 3.2.P.1 - Description and Composition of the Drug Product

- Composition of drug product needs to be described more thoroughly in line with ICH requirements and the Notice to Applicants $(12,13)$.

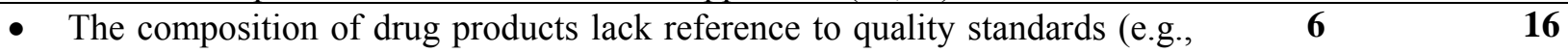
compendial monographs or manufacturer's specifications).

- Description of the drug product (including scoring) needs to be complete.

- GMP certificates supplied for Drug Product Manufactures are not valid (date or lack of import licences for the active substance listed in the GMP certificates).

Other concerns

MODULE 3.2.P.2 - Pharmaceutical Development

- Results from comparative in vitro studies (e.g., dissolution) or comparative in vivo studies (e.g., bioequivalence) should be discussed;

- The discriminatory power of dissolution method as well as the dissolution of different polymorphic forms needs to be submitted;

- Confirmation that the dissolution method will be the same as that used for routine control as well a justification of the dissolution method needs to be submitted;

- Cross references in dossier between - 3.2.P.2.2 and 3.2.P.5.2.2 of the CTD are not correct.

- The choice/amount of excipients and characteristics need to be fully discussed/justified with respect to compatibility and their effects to stability/dosing device;

- The choice/amount of excipients and characteristics need to be fully discussed/justified with respect to pharmaceutical development.

- Justification of lack of in-process controls (IPCs) for a critical parameter of the drug product are lacking;

2816

16

IPCs/screening tests and their use are lacking.

Other concerns

69

MODULE 3.2.P.3 - Manufacture

- A complete description (including details such as holding times of granules, intermediate products and bulk ware), documentation, and results of the validation and/or evaluation studies should be provided for critical steps or critical assays used in the manufacturing process (this includes production scale manufacturing at site of manufacture);

- For validation of analytical procedures, results of the stability of standards and reference solutions should be provided.

- IPCs should be proposed in line with the IPCs specified in the validation protocol;

- Critical steps to ensure the stability should be included as IPCs (eg formal vials leak testing; closure integrity test etc);

- Time schedule of taking the samples for the IPCs should be presented;

- The validation protocol does not identify all critical parameters; 
Table 5. Other Concerns Drug Product .....Continued

- Limits for IPCs should be tightened in line with the results from validation.

- Holding times proposed for intermediate products need to be considered for calculation of the shelf-life;

- Holding times need to be specified in module 3.2.P.3.3 of the CTD.

The manufacturing process should be supplemented with details of 26

12

- Discarding amounts of the drug product (especially for solutions);

- The time between processes (such as the start of the preparation of the solution and its filtration, filter capacity and the maximum duration of filtration);

- Materials used in the manufacture (such as details of membrane filters and the suitability of the filter);

- Critical steps sampling times need to be specified;

- A detailed flow diagram;

- Release specifications amended in compliance with the European Pharmacopeia.

- The batch formula should be adjusted on the basis of assay values;

$21 \quad 10$

- The amounts for active substance and/or excipients; the batch formula specified in module 3.2.P.3.2 of the CTD must equate that in module 2;

- Batch size must be specified (batch size must be in line of the validation studies).

Other concerns

MODULE 3.2.P.4 - Control of Excipients

- $\quad$ For each excipient (including for example capsule shells and printing ink) a

9

20 detailed specification should be given;

- A detailed description of the analytical methods as well as results of the validation should be submitted.

- The certificates of analysis should be provided for each excipient.

- Related substances should be quantified; specificity of the method to suitably separate related substances should be provided.

- Corresponding relevant information on residual solvents present on excipients should be provided.

Other concerns

42

MODULE 3.2.P.5 - Control of Drug Product

- Validation for analytical procedures is inadequate, examples include: 1) residual solvents the HPLC method for related substances lacks the data on LOQ and LOD for the specified impurities, 2) complete validation data on linearity/robustness/accuracy/precision need to be submitted, 3) selectivity of the method for identifying degradants needs to be discussed further;

- Analytical methods should be accompanied by the corresponding detailed European Pharmacopoeia or in-house reference;

- The stability indicating characteristics of all assay and impurity determination methods should be demonstrated;

- Representative chromatograms should be presented.

- Specification(s) for drug product should include those that are applicable for the drug product [deficiencies included the lack of specifications for related substances, codes imprinted on capsules, rubber closures, osmolarity, reconstitution time, a test of "uniformity of dosage units on 
Table 5. Other Concerns Drug Product .... Continued

- film-coated tablet" in line with European Pharmacopoeia monograph 2.9.40 (20)];

- Release and shelf-life specifications should include specific references to analytical methods as listed in sections 2.3.P.5-3 or 3.2.P.5.2-1 in the dossier;

- Limits set for the assay of active substance should be tightened accordingly at shelf-life;

- List of specifications should be dated and should have a version number;

- Release specifications should include an identification test;

- Specifications should clearly identify tests/limits at the release and shelflife.

- Unless otherwise justified, the limit of identified impurities need to be specified;

- The wider stability limits of the above specified impurities do not appear as fully justified;

- Drug product release specification relating to loss on drying should get justified with respect to drug product stability;

- Release tests specifications are not adequately justified based on batch analysis data.

- Characterisation of impurities will need a justification when no testing of polymorphic forms is required;

$35 \quad 12$

- Limits for total related substances need to be tightened in line with degradation products observed at real-time studies.

- The results of batch analyses indicate that levels of impurities are consistently at a high level;

- Certificate of analysis for batches included in the batch analysis need to be submitted;

- Results of related substances in the batch analysis should be presented.

Other concerns

MODULE 3.2.P.6 - Reference Standards or Materials

- Information on the reference standards or reference materials used for

9

69 testing of the drug product should be provided;

- Certificates of analysis should be provided;

- Reference standards used should be standardised against official reference standards when possible.

- In-house working standard IR-spectra confirming structural identity should be provided.

- The use of the reference standard solutions over time should be thoroughly investigated.

- Quality of the reference standard should be established according to the manufacturer of the active substance.

Other concerns

$\mathbf{0}$

MODULE 3.2.P.7 - Container Closure System

- 'Applicants should provide more detailed information regarding the 27 packaging materials for the drug product; 


\section{Table 5. Other Concerns Drug Product .... Continued}

- Information should include the specifications applied by the vendors and quality assurance certificate of compliance as per European Pharmacopoeia Monograph 3.1.4 (10) or Directive 2002/72/EC (11);

- Containers described as 'child resistant' must comply with ISO 8317:2003 (the international standard for child-resistant packaging) (21).

- Since appearance and construction of the primary and secondary container closure system is not clear, the applicant should provide a sample of the finished product;

- Information written in section 6.5 in the SPC is not on accordance with the package presentations contained in the application form and informed about in the dossier;

- It should be clear in the dossier what package presentations are covered by the application.

\section{MODULE 3.2.P.8- Stability}

- The proposed shelf-life cannot be granted yet in view of the limited data; results of stability studies should be presented in an appropriate format (e.g. tabular, graphical, narrative);

- Information on the analytical procedures used to generate the data and validation of these procedures should be included in the dossier;

- For bulk drug product allowed to be stored up to 24 months it should be noted that the expiration period of a production batch should be calculated from the date of release of that batch provided.

- Impurity/degradation shelf-life limits should be tightened from a quality perspective to levels which are actually observed for full scale commercial batches;

- Limits should be proposed for impurities that are based on levels detected in the stability studies.

- Post-approval stability protocols and stability commitments should be submitted in the dossier;

- A commitment that adverse findings of stability data covering the proposed shelf-life for the drug product reported to the European Medicines Agency is required in the dossier.

- Data do not back up the chosen storage conditions.

- Storage requirements should be further justified (for example if semipermeable packaging is utilised, the applicant should discuss if the ICH storage conditions for semi-permeable packaging materials would not be more relevant for the stability studies);

- The shelf life limit for impurities can not be justified based on toxicological data only

\section{ACKNOWLEDGEMENTS.}

Dr. Barbara Van Zwieten Boot, Mr Robert James Hemmings, Mr Michael Chetcuti for their valuable comments during the drafting of the manuscript. Dr. John-Joseph Borg is a full time employee of the Malta Medicines Authority.

\section{REFERENCES}

1. Regulation (EC) No 726/2004 of the European Parliament and of the Council of 31 March 2004 laying down Community procedures for the authorisation and supervision of medicinal products for human and 
veterinary use and establishing a European Medicines Agency. Official Journal of the European Communities, L 136:1-33, 2004.

2. Committee for Medicinal Products for Human Use (CHMP), Guideline on the common technical document for the registration of pharmaceuticals for human use: organisation of common technical document. European Medicines Agency, pp. 1-14, 2004. http:/www.emea.europa.eu/pdfs/human/ich/28799en.p df (accessed 6/8/2009).

3. International Conference on harmonisation of technical requirements for registration of Pharmaceuticals for human Use, "Organisation of the common technical document for registration of pharmaceuticals for human use (M4(R3))", ICH, pp. 1-16, 2004.

http://www.ich.org/LOB/media/MEDIA554.pdf (accessed 6/8/2009).

4. Notice to Applicants (NTA) Volume 2B, in: European Commission, "The Rules governing medicinal Products in the European Union", Brussels, Belgium, pp. 1303, 2006.

5. International Conference on harmonization of technical requirements for registration of Pharmaceuticals for human Use, "The common technical document for the registration of pharmaceuticals for human use: QualityM4Q(R1)”, ICH, pp. 1-24, 2002.

http://www.ich.org/LOB/media/MEDIA556.pdf (accessed 6/8/2009).

6. Committee for Medicinal Products for Human Use (CHMP), Guideline on stability testing of new drug substances and products. European Medicines Agency, pp. $1-20,2003$.

http://www.emea.europa.eu/pdfs/human/ich/273699en. pdf (accessed 6/8/2009).

7. International Conference on harmonization of technical requirements for registration of Pharmaceuticals for human Use, "Stability testing of new drug substances and products Q1A(R2)", ICH, pp1-24, 2003.

http://www.ich.org/LOB/media/MEDIA419.pdf (accessed 6/8/2009).

8. Committee for Medicinal Products for Human Use (CHMP) Guideline on the evaluation of stability data. European Medicines Agency, pp. 1-17, 2003. http://www.emea.europa.eu/pdfs/human/ich/04002en.p df (accessed 6/8/2009).

9. International Conference on harmonization of technical requirements for registration of Pharmaceuticals for human Use, "Evaluation for stability data Q1E", ICH, pp1-19, 2003.

http://www.ich.org/LOB/media/MEDIA415.pdf (accessed 6/8/2009).

10. European Pharmacopoeia monograph on polyolefines, in: European Pharmacopoeia, Strasbourg, France, pp. 274-278, 2007.

11. Directive $2002 / 72 / \mathrm{EC}$ relating to plastic materials and articles intended to come into contact with foodstuffs. Official Journal of the European Communities, L 220: $18-58,2002$.
12. European Directorate for the Quality of Medicines: certification of suitability to monographs of the European Pharmacopoeia top deficiencies found during first assessment of new applications from October 2007 to December 2007, Strasbourg, France, pp 1-4, 2008. http://www.edqm.eu/site/PAPHCEP 08 11_Top_Defic iencies found in Applicationspdf-837-2.html (accessed 6/8/2009).

13. Jeffers, DB., Matthews, BR., Cartwright AC., Defects in applications - an analysis, in Cartwright AC: Matthews BR (eds), Pharmaceutical Product Licensing, Requirements for Europe, $1^{\text {st }}$ ed., by Ellis Horwood, Informa Health Care, New York, NY, pp 190-215, 1991.

14. Committee for Medicinal Products for Human Use (CHMP), Guideline on the Chemistry of New Active Substances. European Medicines Agency, pp. 1-13, 2004.

http://www.emea.europa.eu/pdfs/human/qwp/013096en .pdf (accessed 6/8/2009).

15. Committee for Medicinal Products for Human Use (CHMP), Guideline on the Specification Limits for Residues of Metal Catalysts. European Medicines Agency, pp. 1-34, 2008.

http://www.emea.europa.eu/pdfs/human/swp/444600.pd $\underline{f}$ (accessed 6/8/2009).

16. Committee for Medicinal Products for Human Use (CHMP), Guideline on the impurities: Residual Solvents. European Medicines Agency, pp. 1-24, 1998.

http://www.emea.europa.eu/pdfs/human/ich/028395en. pdf (accessed 6/8/2009).

17. International Conference on harmonization of technical requirements for registration of Pharmaceuticals for human Use, "Impurities: Guideline for residual solvents Q3C(R4)", ICH, pp. 1-25 2009.

http://www.ich.org/LOB/media/MEDIA5254.pdf (accessed 6/8/2009).

18. Committee for Medicinal Products for Human Use (CHMP) Guideline on the Plastic Immediate Packaging Materials. European Medicines Agency, pp. 1-11, 2003.

http://www.emea.europa.eu/pdfs/human/qwp/435903en .pdf (accessed 6/8/2009).

19. Committee for Medicinal Products for Human Use (CHMP), Guideline on the Stability Testing of Existing Active Ingredients and Related Finished Products. European Medicines Agency, pp. 1-18, 2004.

http://www.emea.europa.eu/pdfs/human/qwp/012202en .pdf (accessed 6/8/2009).

20. European pharmacopoeia monograph on Uniformity of Dosage units, in: European Pharmacopoeia, Strasbourg, France, pp. 3370-3374, 2007.

21. International Organization for Standardisation, The international standard for child-resistant packaging (ISO 8317). International Organization for Standardisation. 2003. 